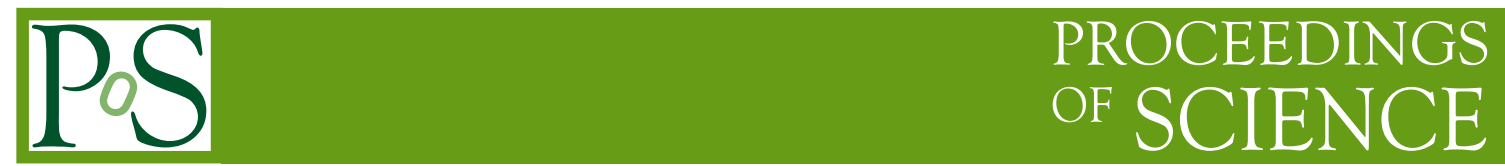

\title{
Light neutral mesons in ALICE
}

\section{Yuri Kharlov*}

Institute for High Energy Physics, Protvino, 142281, Russia

E-mail: Yuri.Kharlov@cern.ch

Light neutral mesons, like $\pi^{0}, \eta$ and $\omega(782)$, will be produced abundantly in the first LHC run in $p p$ collisions. These mesons will be detected by the PHOS detector via their decay channels $\pi^{0} \rightarrow \gamma \gamma, \eta \rightarrow \gamma \gamma$ and $\omega \rightarrow \pi^{0} \gamma$. The physics motivation to study neutral meson production in $p p$ and $A A$ collissions will be discussed in this paper. Acceptances, reconstruction methods, as well as expected $p_{T}$ limits of measured spectra, are presented.

High-pT Physics at LHC -09

February 4-7 2009

Prague, Czech Republic

* Speaker. 


\section{Introduction}

The cross section of inclusive hadron production at large transverse momentum in protonproton and nuclear collisions can be calculated within perturbative quantum chromodynamics (pQCD), because they are produced in fragmentation processes of quarks and gluons issuing from hard parton scattering. As long as an inclusive hadron cross section is calculated in PQCD with the use of the parton density functions of the colliding particles, fragmentation functions and QCD matrix elements, the experimental measurements of hadron production at high $p_{T}$ is a valuable tool for pQCD validation. Therefore, high- $p_{T}$ hadron, along with prompt photons and jets, are considered as sensitive probes for QCD processes at initial stage of hadronic collisions. Hadrons production at high $p_{T}$ in nuclear collisions is sensitive to the properties of strongly interacting matter.

\section{Experimental setup}

Neutral mesons in photonic decay channels will be detected by the ALICE experiment with the use of the photon spectrometer PHOS which is a high-resolution electromagnetic calorimeter made of lead tungstate crystals. The lead tungstate, $\mathrm{PbWO}_{4}$ is one of the heaviest inorganic scintillators with a Moliere radius $R_{M}=2 \mathrm{~cm}$ which defines a tiny lateral granularity and a high spatial resolution of the PHOS detector. The PHOS will consist of 5 modules, each including $56 \times 64$ crystals with a transverse size of $2.2 \times 2.2 \mathrm{~cm}^{2}$. The energy and the coordinate resolutions of the PHOS for photons and electrons is

$$
\frac{\sigma(E)}{E}=\sqrt{\left(\frac{1.3}{E}\right)^{2}+\frac{3.3^{2}}{E}+1.1^{2}}, \quad \sigma(x)=\sqrt{\frac{3.26^{2}}{E}+0.44^{2}} \mathrm{~mm} .
$$

The azimuthal coverage of the PHOS detector will be $220^{\circ}<\varphi<320^{\circ}$ for 5 modules. The partial PHOS geometry available for the first LHC run in 2009 includes 3 modules covering $226^{\circ}<\varphi<$ $320^{\circ}$. The PHOS acceptance in pseudorapidity will be $|\eta|<0.12$.

\section{Cross sections}

Invariant cross section of $\pi^{0}$ meson production in $p p$ collisions can be calculated in the nextto-leading order perturbative QCD (NLO pQCD). This cross section has been calculated using the program INCNLO v1.5 [1]. A parton distribution function set was chosen to be CTEQ5M, the fragmentation function was KKP [2] and a QCD scale $\mu=p_{T}$. The $\pi^{0}$ cross sections in $p p$ collisions at $\sqrt{s}=0.9,5.5,10$ and $14 \mathrm{TeV}$ are shown in Fig.1.

Cross sections of $\eta$ and $\omega(782)$ meson productions can be calculated only in the leading order pQCD, because their fragmentation functions are not known. These cross sections in $p p$ collisions at $\sqrt{s}=0.9,10$ and $14 \mathrm{TeV}$ were obtained in Pythia 6.214 [3] and are shown in Fig.2.

The $\pi^{0}$ production in heavy ion collisions was estimated from that in $p p$ collisions using a binary scaling following the approach [4]:

$$
\sigma\left(A A \rightarrow \pi^{0}\right)=\left\langle N_{\text {coll }}\right\rangle_{C_{1}-C_{2}} \frac{\sigma_{A A}^{\mathrm{geo}}}{\sigma_{N N}} \sigma\left(N N \rightarrow \pi^{0}\right),
$$




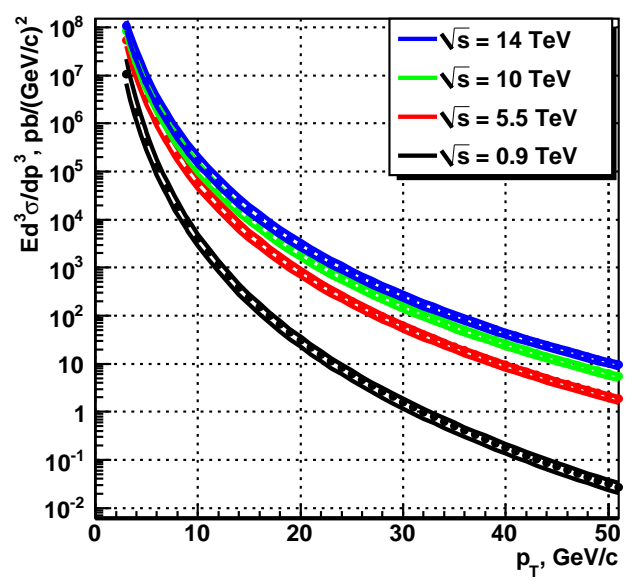

Figure 1: Invariant cross sections of $\pi^{0}$ production in $p p$ collisions at $0.9,5.5,10$ and $14 \mathrm{TeV}$.
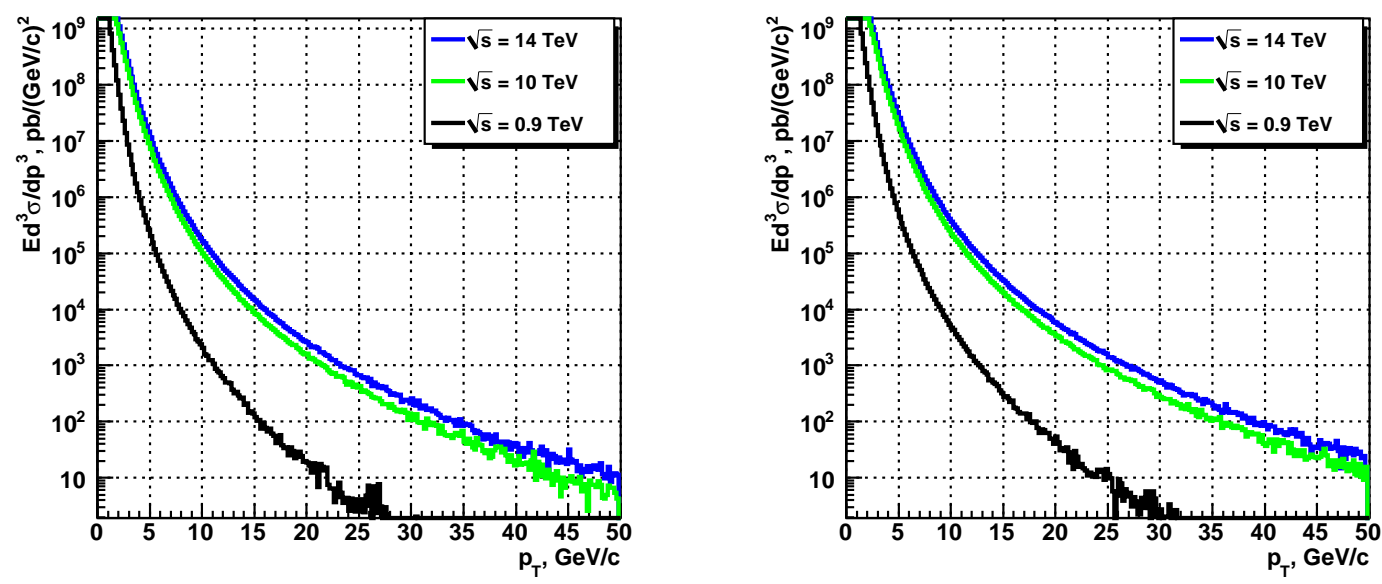

Figure 2: Production cross sections of $\eta$ (left) and $\omega(782)$ (right) in $p p$ collisions at $0.9,10$ and $14 \mathrm{TeV}$.

where $\left\langle N_{\text {coll }}\right\rangle_{C_{1}-C_{2}}$ is the average number of binary nucleus collisions in colliding nuclei at centrality $C_{1}-C_{2}, \sigma_{A A}^{\text {geo }}$ is a geometrical cross section of the nuclei $A A, \sigma_{N N}$ is the total cross section of nucleons, and $\sigma\left(N N \rightarrow \pi^{0}\right)$ is a cross section of $\pi^{0}$ hard production in a nucleon-nucleon collision. For $P b-P b$ collisions and centrality class $0-10 \%$ these values are equal to (see [4], Appendix 1):

$$
\left\langle N_{\text {coll }}\right\rangle_{0-10 \%}=1670, \quad \sigma_{A A}^{\text {geo }}=7745 \mathrm{mb}, \quad \sigma\left(N N \rightarrow \pi^{0}\right)=72 \mathrm{mb} .
$$

The hadron production will be highly suppressed in central heavy ion collisions, and we assume that the nuclear modification factor $R_{A A}$ at the LHC energies will be similar to that at RHIC [5], $R_{A A} \sim 0.2$. The invariant cross section of $\pi^{0}$ production in $P b-P b$ collisions at $5.5 \mathrm{TeV} /$ nucleon and centrality class $0-10 \%$ is shown in Fig.3.

\section{Trigger and run scenario}

The ALICE can operate with different triggers. If the minimum bias trigger partition includes 


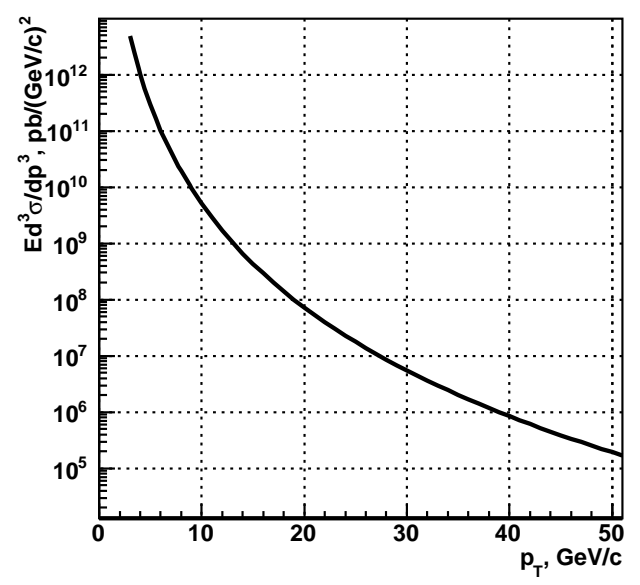

Figure 3: Invariant cross sections of $\pi^{0}$ production in $P b-P b$ collisions at $5.5 \mathrm{TeV} /$ nucleon and centrality class $0-10 \%$.

slower detectors (e.g. TPC), the rate of data taking will be determined by the dead time of these detectors, rather than by the collision rate. The TPC detector is limited by the data taking rate of $200 \mathrm{~Hz}$, which, having in mind a cross section of events detected by the ALICE trigger detectors of $40 \mathrm{mb}$, corresponds to the effective luminosity $\mathscr{L}^{\text {eff }}=5 \cdot 10^{27} \mathrm{~cm}^{-2} \mathrm{~s}^{-1}$.

The PHOS detector can provide its own L0 trigger defined by the energy deposited in any patch of adjacent $2 \times 2$ crystals above a threshold. At the low enough threshold, $200-500 \mathrm{MeV}$, the rate of events triggered by the PHOS L0 trigger becomes rather low, at the level of a $100-200 \mathrm{~Hz}$ which is below the PHOS intrinsic data taking rate. If the PHOS is operating in a standalone trigger partition, the data taking rate is solely determined by the beam luminosity.

The first LHC run with proton-proton collisions is expected at the energy $\sqrt{s}=10 \mathrm{TeV}$ and a lower luminosity $\mathscr{L}=5 \cdot 10^{28} \mathrm{~cm}^{-2} \mathrm{~s}^{-1}$ (see, e.g. [6]). The nominal luminosity for the ALICE experiment is $\mathscr{L}=3 \cdot 10^{30} \mathrm{~cm}^{-2} \mathrm{~s}^{-1}$. The run time can vary from weeks to months with some duty factor. To have more definitive predictions, we calculate integrated luminosity for several periods of data taking: 3 day, 30 days and 3 months which are collected in Table 1. Keeping in mind

\begin{tabular}{c|c|c|c}
\hline \multirow{2}{*}{$T$} & \multicolumn{3}{|c}{$\mathscr{L}, \mathrm{cm}^{-2} \mathrm{~s}^{-1}$} \\
\cline { 2 - 4 } & $5 \cdot 10^{27}$ & $5 \cdot 10^{28}$ & $3 \cdot 10^{30}$ \\
\hline 3 days & 1.3 & 13 & 780 \\
\hline 30 days & 13 & 130 & 7800 \\
\hline 3 months & 39 & 390 & $2.2 \cdot 10^{4}$ \\
\hline
\end{tabular}

Table 1: Integrated luminosity $\int \mathscr{L} d T$ in $\mathrm{nb}^{-1}$ for different LHC luminosities $\mathscr{L}$ and different data taking times $T$.

the aforementioned run scenarios, we estimate the detection rate of light mesons for integrated luminosities $\int \mathscr{L} d T=10,100$ and $300 \mathrm{nb}^{-1}$.

The heavy ion run will have the $P b-P b$ collision energy $5.5 \mathrm{TeV} /$ nucleon and the luminosity $\mathscr{L}=5 \cdot 10^{26} \mathrm{~cm}^{-2} \mathrm{~s}^{-1}$. Expected duration of the heavy ion run is about $10^{6} \mathrm{~s}$, which will give the 
integrated luminosity $\int \mathscr{L} d T=0.5 \mathrm{nb}^{-1}$.

\section{Detection of neutral mesons in PHOS}

The geometrical acceptance for reactions $\pi^{0} \rightarrow \gamma \gamma, \eta \rightarrow \gamma \gamma$ and $\omega(782) \rightarrow \pi^{0} \gamma \rightarrow 3 \gamma$ was calculated in Monte Carlo simulations for 3 adjacent PHOS modules corresponding the PHOS configuration available for the first LHC run. The parameterization of the acceptance $A\left(p_{T}\right)$ as a function of the meson's transverse momentum $p_{T}$ normalized to the unit rapidity $|y|<0.5$ and to the full azimuth angle $\Delta \phi=2 \pi$ can be expressed by the formula

$$
A\left(p_{T}\right)=\left(a+b p_{T}\right)\left(1-\exp \frac{c-p_{T}}{d}\right)
$$

and shown in Fig.4 (left) with parameters given in the table (right).

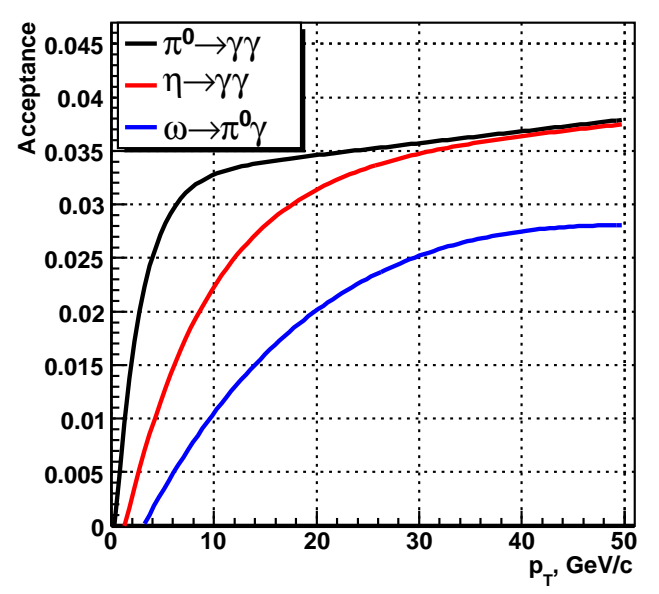

\begin{tabular}{c|c|c|c|c}
\hline Reaction & $a$ & $b$ & $c$ & $d$ \\
\hline$\pi^{0} \rightarrow \gamma \gamma$ & 0.032 & $1.1 \cdot 10^{-4}$ & 0.355 & 2.51 \\
$\eta \rightarrow \gamma \gamma$ & 0.033 & $9.1 \cdot 10^{-5}$ & 1.33 & 8.10 \\
$\omega \rightarrow \pi^{0} \gamma$ & 0.183 & $2.0 \cdot 10^{-3}$ & 2.37 & 120 \\
\hline
\end{tabular}

Figure 4: Acceptance parameterization for $\pi^{0}, \eta$ and $\omega(782)$ in 3 PHOS modules.

Reconstruction of neutral mesons in PHOS is performed using the invariant mass spectrum of 2 reconstructed clusters for 2-photon decay channels of $\pi^{0}$ and $\eta$ or of 3 reconstructed clusters for $\omega \rightarrow \pi^{0} \gamma$. Due to a low detector occupancy in $p p$ collisions, the $\pi^{0}$ peak reveals itself without applying any particle identification criteria for reconstructed clusters. The invariant mass spectrum of cluster pairs in the mass range around $\pi^{0}$ is illustrated in Fig. 5 for two $p_{T}$ bins, $0-1 \mathrm{GeV} / \mathrm{c}$ (left) and 3-4 GeV/c (right). These spectra were obtained in the full Monte Carlo simulations of 4 million Pythia minimum bias events in the ALICE setup. The invariant mass spectra are fitted by the sum of the Gaussian and the polynomial of the first order, represented by the red curve. The number of reconstructed $\pi^{0}$ can be extracted from the invariant mass spectra by integrating the Gaussian part of the fitting function. The raw $p_{T}$ spectrum of reconstructed $\pi^{0}$ per one $p p$ collisions as a function of $p_{T}$ is shown in Fig.6 (left). The ratio of the number of reconstructed $\pi^{0}$ to the combinatorial background is given in Fig.6 (right). The available simulated statistics is not enough to reconstruct $\eta \rightarrow \gamma \gamma$ and $\omega \rightarrow \pi^{0} \gamma$, but the reconstruction techniques should be similar to that of $\pi^{0}$. The obtained raw spectrum should be converted to the production cross section via 

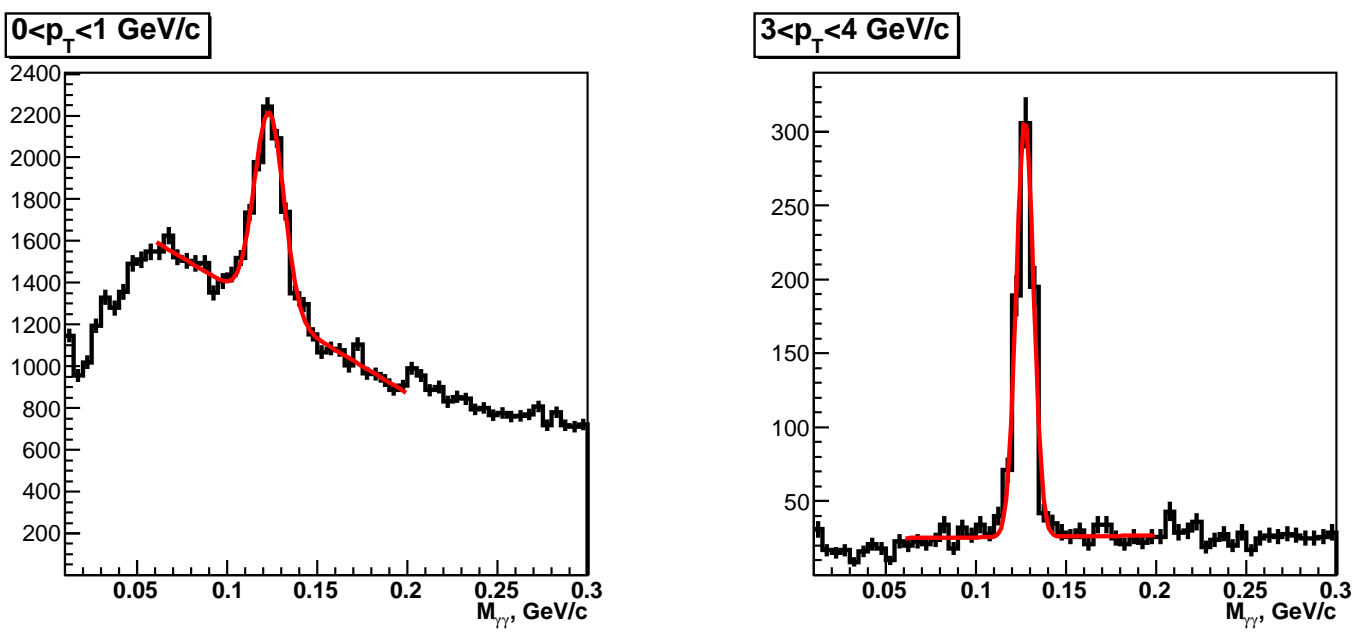

Figure 5: Invariant mass spectrum of cluster pairs reconstructed in PHOS in $p p$ collisions at $\sqrt{s}=10 \mathrm{TeV}$ at different $p_{T}$.
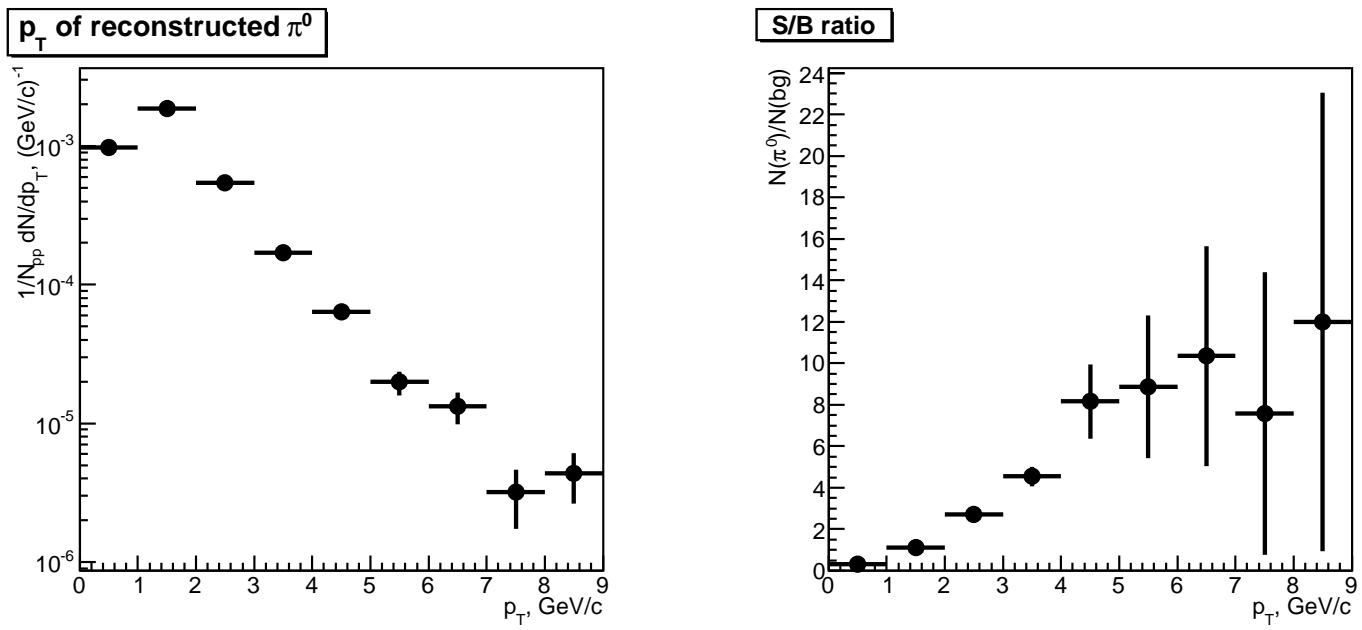

Figure 6: The raw $p_{T}$ spectrum of reconstructed $\pi^{0}$ in $p p$ collisions (left) and a ratio of signal to background (right).

correcting it by the acceptance $A$ (Fig.4), the reconstruction and trigger efficiencies $\varepsilon$, and by the off-vertex background $c_{\text {offvtx }}$ :

$$
\frac{d \sigma}{d p_{T}}=\frac{d N}{d p_{T}} \frac{1}{\mathscr{L} \cdot T \cdot A \cdot \varepsilon \cdot c_{\mathrm{offvtx}}} .
$$

The reconstruction efficiency of one photon is $\varepsilon_{\gamma}=0.85$ [7], and for $N$-photon final states it is estimated as $\varepsilon_{\gamma}^{N}$. The off-vertex background was estimated at the level less then $0.1 \%$.

Detection rate can be estimated from the predicted cross sections (Figs.1 and 2) by applying the inverse relation between the cross section and event rate (5.1). Detection rate of $\pi^{0}$ in $p p$ collisions at $\sqrt{s}=10 \mathrm{TeV}$ and for three integrated luminosities $\int \mathscr{L} d T=10,100$ and $300 \mathrm{nb}^{-1}$ is shown in Fig.7 (left). The number of detected $\pi^{0}$ in the central $P b-P b$ collision at $\sqrt{s}=5.5 \mathrm{TeV} /$ nucleon at centrality class $0-10 \%$ and for the integrated luminosity $\int \mathscr{L} d T=0.5 \mathrm{nb}^{-1}$ is presented in Fig.7 
(right). The number of detected $\eta$ and $\omega$ mesons in the photonic decay channels was calculated for
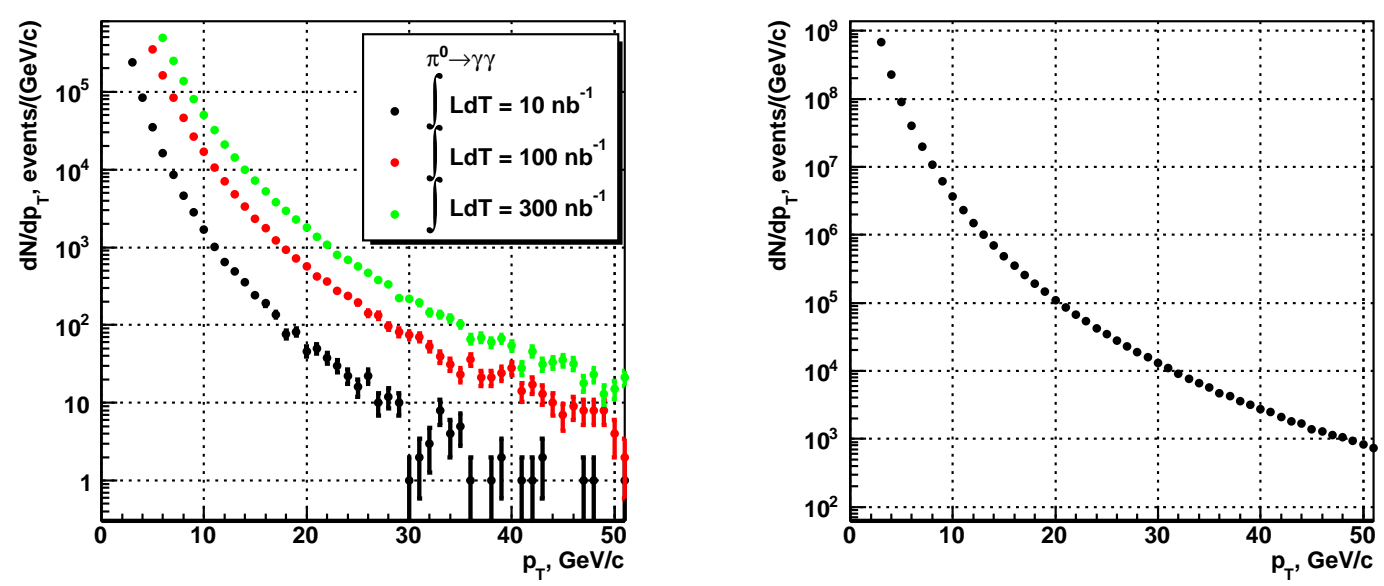

Figure 7: Detection rate of $\pi^{0}$ in 3 PHOS modules in $p p$ collisions at $\sqrt{s}=10 \mathrm{TeV}$ (left) and in central $P b-P b$ collisions at $\sqrt{s}=5.5 \mathrm{TeV} /$ nucleon (right).

$p p$ collisions only, because their detection in high-multiplicity environment in $\mathrm{Pb}-\mathrm{Pb}$ collisions seems to be a very challenging task. Detection rate of these mesons for three integrated luminosities are illustrated by Fig.8.
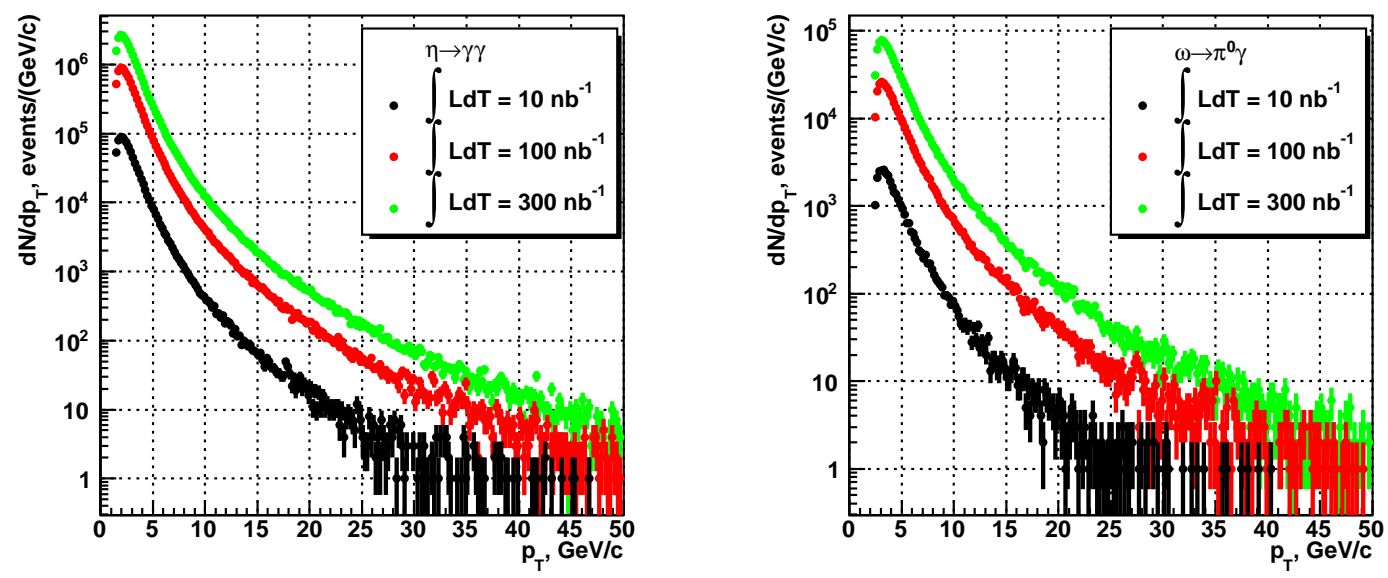

Figure 8: Detection rate of $\eta$ (left) and $\omega(782)$ (right) in $p p$ collisions at $10 \mathrm{TeV}$.

\section{Summary}

The ALICE experiment will measure inclusive spectra of light neutral mesons in $p p$ and $P b-$ $P b$ collisions in a wide transverse momentum range. The first data obtained from the $p p$ run of LHC will provide enough statistics to measure spectra of $\pi^{0}, \eta$ and $\omega(782)$ at transverse momenta up to $p_{T}=30-50 \mathrm{GeV} / \mathrm{c}$. Precise measurement of these spectra can be used to validate the 
pQCD calculations. The detection of neutral mesons in $P b-P b$ collisions will give the nuclear modification factor $R_{A A}$ and will help for interpretation of the parton energy loss models.

\section{References}

[1] P. Aurenche, M. Fontannaz, J. P. Guillet, B. A. Kniehl and M. Werlen, Eur. Phys. J. C 13,347 (2000), http://wwwlapp.in2p3.fr/lapth/PHOX_FAMILY.

[2] B. A. Kniehl, G. Kramer and B. Potter, Nucl. Phys. B 582 (2000) 514 [arXiv:hep-ph/0010289].

[3] T. Sjostrand, S. Mrenna and P. Skands, JHEP 0605 (2006) 026 [arXiv:hep-ph/0603175].

[4] F.Arleo et al., Photon physics in heavy ion collisions at the LHC. hep-ph/0311131. In: Hard Probes in Heavy Ion Collisions at the LHC Book, pages 367-493.

[5] A. Adare et al. [PHENIX Collaboration], Phys. Rev. Lett. 101 (2008) 232301 [arXiv:0801.4020 [nucl-ex]].

[6] R.Bailey, In: Proceedings of Chamonix 2009 workshop on LHC Performance (2009), 23. https://espace.cern.ch/acc-tec-sector/Chamonix/Chamx2009/html/session.htm

[7] ALICE Collaboration et al. J. Phys. G: Nucl. Part. Phys. 32 (2006) 1295-2040. 